\title{
Flaxseed oil supplementation manipulates correlations between serum individual mol \% free fatty acid levels and insulin resistance in type 2 diabetics. Insulin resistance and percent remaining pancreatic $\beta$-cell function are unaffected
}

\author{
${ }^{1}$ Barre DE, ${ }^{2}$ Mizier-Barre KA, ${ }^{3}$ Griscti O, ${ }^{4}$ Hafez K \\ ${ }^{1}$ Department of Health Sciences and Emergency Management, Cape Breton University, Sydney, Nova Scotia, Canada; \\ ${ }^{2}$ Department of Biology, Cape Breton University, Sydney, Nova Scotia, Canada; \\ ${ }^{3}$ School of Nursing, Cape Breton University, Sydney, Nova Scotia, Canada; \\ ${ }^{4} \mathrm{Dr}$ Soliman Faqeeh Hospital/King Abdulla University of Science and Technology, Jeddah, Saudi Arabia \\ E-mail:ed_barre@cbu.ca
}

Objectives. Elevated total serum free fatty acids (FFAs) concentrations have been suggested, controversially, to enhance insulin resistance and decrease percent remaining $\beta$-cell function. However, concentrations of individual serum FFAs have never been published in terms of their relationship (correlation) to homeostatic model assessment-insulin resistance (HOMA-IR) and percent remaining $\beta$-cell function $(\mathrm{HOMA}-\% \beta$ ) in the type 2 diabetics (T2Ds). Alpha-linolenic acid consumption has a negative correlation with the insulin resistance, which in turn is negatively correlated with the remaining $\beta$-cell function. The primary objective was to test the hypothesis that there would be different relationship (correlation) between the blood serum individual free FFA mol \% levels and HOMA-IR and/or HOMA- $\% \beta$ in T2D. The secondary objective was to test the hypothesis that flaxseed oil, previously being shown to be ineffective in the glycemic control in T2Ds, may alter these correlations in a statistically significant manner as well as HOMA-IR and/ or HOMA-\% $\%$.

Methods. Patients were recruited via a newspaper advertisement and two physicians have been employed. All the patients came to visit one and three months later for a second visit. At the second visit, the subjects were randomly assigned (double blind) to flaxseed or safflower oil treatment for three months, until the third visit.

Results. Different statistically significant correlations or trends towards among some serum individual free FFA mol \% levels and HOMA-IR and HOMA- $\% \beta$, pre- and post-flaxseed and safflower oil supplementation were found. However, flaxseed oil had no impact on HOMA-IR or HOMA-\% $\beta$ despite statistically significant alterations in correlations compared to baseline HOMA-IR.

Conclusions. The obtained data indicate that high doses of flaxseed oil have no statistically significant effect on HOMA-IR or HOMA- $\% \beta$ in T2Ds, probably due to the additive effects of negative and positive correlations.

Key words: insulin resistance, pancreatic $\beta$-cell function, free fatty acids, alpha-linolenic acid, flaxseed oil, type 2 diabetes

Corresponding author: Dr Ed. Barre, Department of Health Sciences and Emergency Management, Cape Breton University, P.O. Box 5300, Sydney, Nova Scotia, B1P-6L2 Canada; phone: (902) 563-1921; fax: (902) 563-1945; e-mail: ed_barre@cbu.ca. 
Insulin resistance and $\beta$-cell function, both key components of the management of the type 2 diabetes (T2D), may be assessed by homeostatic model assessment-insulin resistance (HOMA-IR) and homeostatic model assessment-percentage beta cell function (HOMA-\% $\beta$ ) (Wallace et al. 2004).

Elevated concentrations of serum total free fatty acids (FFAs) have been suggested, albeit controversially, to enhance insulin resistance and decrease the $\beta$-cell function (Reaven and Chen 1988; Eriksson et al. 1991; Butler et al. 2001; Blaak 2003; Hawkins et al. 2003; Moore et al. 2004; Robertson et al. 2004; Poitout et al. 2006; Delarue and Magnan 2007; Ye 2007; Poitout and Robertson 2008; Poitout et al. 2010; Boden 2011; Karpe et al. 2011; Morita et al. 2012; Salgin et al. 2012).

However, mol \% levels of the blood serum of the individual FFAs have never been studied for their relationships (correlations) to HOMA-IR or HOMA- $\% \beta$ either under basal conditions or after a treatment in the type 2 diabetics (T2Ds). The controversial impact of the serum, total individual FFA pool may be a reflection of the differential fatty acid (FA) compositions of the pool in different studies.

As different FAs have different lengths, positional (omega) and geometric (cis, trans) isomers, and hence structure, it is reasonable to suggest that they may differentially influence the insulin resistance and/or $\beta$-cell function. Furthermore, as dietary FA consumption changes, one may find alterations in the relationship of the individual FFAs to each of insulin resistance and pancreatic $\beta$-cell function (Newens et al. 2011). More specifically, alpha-linolenic acid consumption (ALA, 18:3n-3) has been proposed to reduce insulin resistance in human $\mathrm{T} 2 \mathrm{D}$ (Wang et al. 2013) albeit prior to the current study. Generally, the relationship, if any exists, between ALA and pancreatic beta cell function in T2Ds is not clear. However, Armoni et al. (2005) have found elevated concentrations of serum free palmitic acid (PA, 16:0), stearic acid (SA 18:0), oleic acid (OA, 18:1 n-9) linoleic acid (LA, 18:2 n-6), ALA, and arachidonic acid (AA, 20:4n6) relative to healthy controls, while the mol \% levels were similar though perhaps inferred to be connected to higher HOMA-IR and lower HOMA-\% $\%$. However, the data of other studies have demonstrated differing relationships/correlations between the varying mol \% levels of serum/plasma individual FFAs and HOMAIR and/or HOMA-\% $\beta$ (Yi et al. 2007; Liu et al. 2010; Grapov et al. 2012).

There is an interest in omega 3 FAs to control the blood glucose levels. However, several studies in the 1980s showed a deterioration of glycemic control in patients with T2D consuming high doses of fish oil containing eicosapentaenoic acid (EPA, 20:5 n-3) and docosahexaenoic acid (DHA, 22:6 n-3) or purified EPA or DHA (Glauber et al. 1998; Woodman et al. 2002; Nettleton and Katz 2005). However, lower doses of fish oil or purified EPA or DHA resulted in no change in the glycemic control (Rivellese et al. 1996; Nakamura et al. 1998; Montori et al. 2000; Nettleton and Katz 2005). Low doses of flaxseed oil containing ALA did not improve glycemic control in T2Ds. McManus et al. (1996) and Goh et al. (1997) giving $35 \mathrm{mg} \mathrm{ALA} / \mathrm{kg}$ body weight/day in the form of flaxseed oil for three months, failed to note any change in the fasting blood plasma glucose. Taylor et al. (2010) and Barre et al. (2005a,b; 2008) also failed to show an impact of flaxseed oil on glucose management in T2Ds, even with much higher doses of flaxseed oil used (81.5 and $60 \mathrm{mg} \mathrm{ALA} / \mathrm{kg}$ body weight /day, respectively). As ALA is slowly desaturated and elongated to EPA and DHA, it was reasoned that higher doses of ALA should be examined, given that high doses of flaxseed oil dramatically reduce platelet reactivity (Barre et al. 2005a,b). This suggests a significant accumulation of bioactive EPA and DHA, thus rationalizing the current study on high dose ALA. Such information is important to researchers, physicians, and T2D patients dealing with the impact of flaxseed oil on the glucose management by high dose flaxseed oil in T2Ds.

The primary objective of this work was to test the hypothesis that there would be different relationships (correlations) between the blood serum individual FFA mol \% levels and each of HOMA-IR and/or HOMA-\% $\beta$ in human T2D. The secondary objective was to test the hypothesis that flaxseed oil (rich in ALA) supplementation could alter these correlations and consequently HOMA-IR and/or HOMA- $\% \beta$ in comparison with the placebo safflower oil (poor in ALA). This is the first study examining the potential relationships between serum individual FFA mol \% levels and each of HOMA-IR and HOMA- $\% \beta$ in human T2D. This study builds on the already published data, including data in Tables 1 and 2, and parts of Tables 3 and Table 8 (Barre et al. 2008).

\section{Materials and Methods}

Subjects. The inclusion criteria were: 18 years of age or older, T2D and not involvement in the physical training program, not taking insulin or any omega 3 supplement including fish oil, not pregnant or planning becoming to be pregnant and not to have contraindication to flaxseed oil or safflower oil. The exclusion 
criteria were: those not meeting the inclusion criteria and those with hepatic or kidney disease. Patients were recruited via a Sydney, Nova Scotia newspaper advertisement and two area physicians. From 40 patients (20 males, 20 females) who attended visit 1 , 18 completed visits 1, 2 and the flaxseed oil intervention, and 14 completed visits 1,2 and the safflower oil intervention. Eighteen males and 14 females completed this study. This study received approval from the Cape Breton University Human Ethics Review Committee and was conducted in accordance with the Declaration of Helsinki. Written informed consent was obtained from all study participants.

Management protocol. The experimental design is outlined in Figure 1. Subjects came fasted (12-14 h) for the first visit, at which the time of the study was explained before they were consented. Three months later, patients came for the second visit. After these two visits, the subjects continued with their normal daily activities without intervention. At the 2 nd visit, the patients were randomized into ALA rich $(\sim 57.2$ weight \%) flaxseed oil (60 mg ALA/kg body weight/ day) or ALA poor ( $<0.1$ weight $\%$ ) safflower oil in the form of $1 \mathrm{~g}$ gelatin capsules for three months until the 3rd visit (all patients consumed approximately $103 \mathrm{mg}$ of oil/ $\mathrm{kg}$ body weight/day). On all the visits, the age and sex of participants were noted, and body weight and height as well as body mass index (BMI) were determined. Diet, exercise, smoking, and medication records were kept by the patients throughout the study and collected at each visit.
The study design allowed no intervention for three month which allowed to establish the stability of fasting serum glucose (FSG), and insulin concentrations (FSI) (Barre et al. 2008). The intervention during the following three months required random intervention with flaxseed oil or placebo (safflower oil). Accurate assessment of the impact of flaxseed oil on HOMA-IR and HOMA- $\% \beta$ required three-month period. The extent of changes, if any, in the control group compared to those in the treated group allowed a decision on whether the flaxseed oil dose had any impact on HOMA-IR and/or HOMA-\% $\beta$. Blood was withdrawn into redtop vacutainers and allowed to clot for $45 \mathrm{~min}$ at room temperature before spinning at $1000 \mathrm{xg}$ for $15 \mathrm{~min}$. Serum was removed by transfer pipet being careful not disturbed the packed cells.

Free fatty acid extraction. Serum FFAs were determined by first taking $2.0 \mathrm{ml}$ of serum (that had been frozen at $-80^{\circ} \mathrm{C}$ until analysis), standards (dissolved in n-heptane) or UHP water (for use as blank) and spiking each with $50 \mu \mathrm{l}$ of $500 \mathrm{mM}$ 17:0 (in n-heptane) as an internal standard. Then $0.5 \mathrm{ml}$ spiked serum standard, samples and blanks were extracted using $2.5 \mathrm{ml}$ of isopropanol-n-heptane-phosphoric acid (2 M) (40:10:1 v/v), vortexed, sonicated, vortexed, and then $1 \mathrm{ml}$ of $\mathrm{n}$-heptane and $1.5 \mathrm{ml}$ UHP water added. Then the spiked blanks, standards, and samples were vortexed and sonicated followed by centrifugation at $1000 \times \mathrm{g} 10 \mathrm{~min}$ at $4{ }^{\circ} \mathrm{C}$. The top layer $(1.5 \mathrm{ml})$, with isolated FFAs (Mehta et al. 1998) was removed and dried under nitrogen at $100^{\circ} \mathrm{C}$.

Visit 1 - patients consent and give a blood sample; patients instructed to keep records of diet (including alcohol), exercise, medication (type and dose of each drug) and smoking patterns. Values for all parameters (weight, height, plasma insulin, glucose, HOMA-IR, HOMA-\% (mol \% free fatty acids are determined for visit 1).

$$
\downarrow
$$

3 months, no intervention with flaxseed oil or safflower oil (patients otherwise carry on with normal life patterns)

Visit 2 - patients return and give a blood sample; records are collected. Values for all parameters indicated above are averaged for visits 1 and 2

$$
\begin{aligned}
& 3 \text { months, intervention with flaxseed oil or safflower oil (patients randomly assigned to } \\
& \text { flaxseed oil or safflower oil but otherwise carry on with normal life patterns) }
\end{aligned}
$$

Visit 3 - patients return and give a blood sample; records are collected. Values for all parameters indicated above are determined.

Figure 1. Flow chart of patient visits and interventions. 
HPLC separation of FFAs. Individual FFAs were then derivatised with phenacyl bromide using $\beta$-bromoacetophenone, triethylamine and acetic acid (Mehta et al. 1998). Samples, standards and blanks were then dried down under nitrogen in a dry block at $100^{\circ} \mathrm{C}$ and reconstituted with $200 \mu \mathrm{l}(166 \mu \mathrm{l}$ of acetonitrile first followed by $34 \mu \mathrm{l}$ of water) of mobile phase (acetonitrile:water, 83:17 v/v). Samples, standards and blanks were then vortexed and centrifuged to remove any particulate matter, the supernatant removed and mixed with $600 \mu \mathrm{l}$ of mobile phase. Fatty acid analysis was done by HPLC using a Waters Resolve C18 $5 \mu \mathrm{m}$ $3.9 \times 300 \mathrm{~mm}$ column, a Waters 717 Plus autosampler, 600 pump and controller and a Waters 2487 UV/VIS detector. The injection volume was $100 \mu \mathrm{l}$ of mobile phase containing the derivatised FAs with a mobile phase flow rate of $2 \mathrm{ml} / \mathrm{min}$ for $5 \mathrm{~h}$ with $242 \mathrm{~nm}$ detection. Peaks were identified by comparison with retention time of certified FA standards that had been derivatised with phenacyl bromide as above (Mehta et al. 1998). Mol \% FFAs were calculated.

Glucose, insulin, HOMA-IR and HOMA-\% $\beta$. Glucose was measured by a C2 kit (Wako, Richmond, Virginia, USA) and serum insulin measured by human insulin ELISA kit (Linco, St. Charles,

Table 1

Fatty acid composition (weight percent), i.e. mg of an individual fatty acid per $100 \mathrm{mg}$ of fatty acids in flaxseed oil (treatment) and safflower oil (placebo)

\begin{tabular}{ccc}
\hline Fatty acid & Flaxseed oil & Safflower oil \\
\hline $14: 0$ & - & 0.2 \\
$16: 0$ & 5.6 & 6.3 \\
$18: 0$ & - & 2.2 \\
$18: 1 \mathrm{n}-9$ & 15.1 & 14.1 \\
$18: 2 \mathrm{n}-6$ & 14.6 & 74.2 \\
$18: 3 \mathrm{n}-3$ & 57.2 & $<0.1$ \\
\hline $20: 4 \mathrm{n}-6$ & - & - \\
\hline $20: 5 \mathrm{n}-3$ & - & - \\
\hline $22: 6 \mathrm{n}-3$ & - & - \\
\hline
\end{tabular}

Table 2

Oil and alpha-linolenic acid consumption in human type 2 diabetic patients

\begin{tabular}{lcc}
\hline Consumption & Flaxseed oil & Safflower oil \\
\hline Total oil (g/day) & $9.6 \pm 0.3$ & $9.5 \pm 0.3$ \\
Total oil (mg/kg/day) & $105.3 \pm 0.8$ & $103.2 \pm 1.2$ \\
Alpha-linolenic acid (g/day) & $5.4 \pm 0.2$ & $<0.01$ \\
Alpha-linolenic (mg/kg/day) & $60.0 \pm 0.5$ & $<0.01$ \\
\hline
\end{tabular}

Missouri, USA) according to the manufacturer's directions. The glucose and insulin values have been published previously (Barre et al. 2008).

HOMA-IR was calculated by multiplying FSG $(\mathrm{mmol} / \mathrm{l})$ by FSI $(\mathrm{mU} / \mathrm{L})$ and dividing the product by 22.5 while HOMA- $\% \beta$ is determined by the formula $(20 \times \mathrm{FSI}) /(\mathrm{FSG}-3.5)$ (Matthews et al. 1985). In both formulae, glucose was measured in $\mathrm{mmol} / \mathrm{L}$ and insulin in $\mathrm{mU} / \mathrm{L}$.

Statistical analysis. Power was 0.80 to see a difference of $1 \mathrm{mmol}$ of glucose/1 using 12 subjects per group with $\mathrm{p}<0.05$. Males and females were combined to give sufficient statistical power. For a given parameter (FFAs, insulin, glucose, HOMA-IR and HOMA-\% $\beta$ ) and person, visits 1 and 2 were averaged. A general linear model analysis (repeated measures) was performed with a significance level of $\mathrm{p}<0.05$. Pearson correlations were done between each of the averaged (for visits 1 and 2) baseline serum individual FFA mol \% levels and averaged visit 1 and 2 values for each of each of HOMA-IR and HOMA-\% $\beta$ (Table 4). Pearson correlations were done also between each of flaxseed oil and safflower oil-driven consumptiondriven individual FFA levels and each of HOMA-IR and HOMA- $\% \beta$ (visit 3) (Table 5 and Table 6, respectively) (Roscoe 1975).

\section{Results}

Composition of flaxseed and safflower oils is found in Table 1. The single greatest level of FA in the flaxseed oil is ALA.

Oil and ALA consumption in $\mathrm{g} / \mathrm{day}$ and $\mathrm{mg} / \mathrm{kg}$ body weight/day for each of flaxseed oil, safflower oil, and ALA is found in Table 2. Oil mass consumption was the same between flaxseed and safflower oil groups, though ALA consumption was much higher in the flaxseed oil consumers. Diet, smoking status, exercise and medications (type, dose) were consistent throughout the study.

Table 3 reveals similarity of patient entry characteristics between those assigned to flaxseed and safflower oil groups (a two sample t-test showed no statistically significant difference between the prevalues for each of BMI, plasma individual FFAs, glucose, insulin, HOMA-IR and HOMA- $\% \beta$ when flaxseed oil and safflower oil groups were compared.

Table 4 shows the baseline correlations between each of the individual serum FFAs and each of HOMA-IR and HOMA- $\% \beta$ variables averaged for visits 1 and 2 . The only significant correlation pretreatment or placebo is the positive correlation of PA with HOMA-IR with trends $(\mathrm{p}<0.2)$ toward a posi- 
Table 3

Pre-treatment characteristics of subjects (all Caucasian)

\begin{tabular}{|c|c|c|c|}
\hline Parameter & & Flaxseed oil & Safflower oil \\
\hline Age (years) & & $59.5 \pm 1.7$ & $60.7 \pm 2.9$ \\
\hline $\mathrm{N}$ & & $\begin{array}{c}18 \\
\text { (10 males, } \\
8 \text { females })\end{array}$ & $\begin{array}{c}14 \\
\text { (8 males, } \\
6 \text { females) }\end{array}$ \\
\hline $\begin{array}{l}\text { BMI } \\
\left(\mathrm{kg} / \mathrm{m}^{2}\right)\end{array}$ & $\begin{array}{l}\text { Visit } 1 \\
\text { Visit } 2\end{array}$ & $\begin{array}{l}32.4 \pm 0.9 \\
32.2 \pm 1.0\end{array}$ & $\begin{array}{l}30.3 \pm 0.7 \\
30.3 \pm 0.8\end{array}$ \\
\hline $\begin{array}{l}\text { Glucose } \\
(\mathrm{mmol} / \mathrm{l})\end{array}$ & $\begin{array}{l}\text { Visit } 1 \\
\text { Visit } 2\end{array}$ & $\begin{array}{l}8.6 \pm 0.8 \\
8.0 \pm 0.8\end{array}$ & $\begin{array}{l}8.1 \pm 0.8 \\
7.8 \pm 0.7\end{array}$ \\
\hline $\begin{array}{l}\text { Insulin } \\
(\mathrm{mU} / \mathrm{ml})\end{array}$ & $\begin{array}{l}\text { Visit } 1 \\
\text { Visit } 2\end{array}$ & $\begin{array}{l}12.4 \pm 1.5 \\
13.1 \pm 2.3\end{array}$ & $\begin{array}{l}8.4 \pm 1.2 \\
8.3 \pm 1.2\end{array}$ \\
\hline HOMA-IR & $\begin{array}{l}\text { Visit } 1 \\
\text { Visit } 2\end{array}$ & $\begin{array}{l}4.8 \pm 0.7 \\
4.7 \pm 1.1\end{array}$ & $\begin{array}{l}3.0 \pm 0.6 \\
2.9 \pm 0.6\end{array}$ \\
\hline HOMA- $\% \beta$ & $\begin{array}{l}\text { Visit } 1 \\
\text { Visit } 2\end{array}$ & $\begin{array}{c}48.6 \pm 8.0 \\
58.8 \pm 11.1\end{array}$ & $\begin{array}{l}36.5 \pm 5.5 \\
38.6 \pm 7.9\end{array}$ \\
\hline $12: 0$ & $\begin{array}{l}\text { Visit } 1 \\
\text { Visit } 2\end{array}$ & $\begin{array}{c}0.81 \pm 0.09 \\
1.15 \pm 0.2\end{array}$ & $\begin{array}{l}0.81 \pm 0.09 \\
0.90 \pm 0.07\end{array}$ \\
\hline 14:0 & $\begin{array}{l}\text { Visit } 1 \\
\text { Visit } 2\end{array}$ & $\begin{array}{l}3.1 \pm 0.2 \\
2.8 \pm 0.1\end{array}$ & $\begin{array}{l}2.6 \pm 0.2 \\
2.6 \pm 0.2\end{array}$ \\
\hline $14: 1 \mathrm{n}-5$ cis & $\begin{array}{l}\text { Visit } 1 \\
\text { Visit } 2\end{array}$ & $\begin{array}{l}0.10 \pm 0.06 \\
0.11 \pm 0.03\end{array}$ & $\begin{array}{l}0.05 \pm 0.01 \\
0.14 \pm 0.05\end{array}$ \\
\hline $14: 1 \mathrm{n}-5$ trans & $\begin{array}{l}\text { Visit } 1 \\
\text { Visit } 2\end{array}$ & $\begin{array}{l}0.15 \pm 0.02 \\
0.14 \pm 0.02\end{array}$ & $\begin{array}{l}0.20 \pm 0.07 \\
0.07 \pm 0.02\end{array}$ \\
\hline $16: 0$ & $\begin{array}{l}\text { Visit } 1 \\
\text { Visit } 2\end{array}$ & $\begin{array}{l}23.1 \pm 0.9 \\
21.2 \pm 0.8\end{array}$ & $\begin{array}{l}22.8 \pm 0.8 \\
20.7 \pm 0.7\end{array}$ \\
\hline $16: 1 \mathrm{n}-7$ cis & $\begin{array}{l}\text { Visit } 1 \\
\text { Visit } 2\end{array}$ & $\begin{array}{l}6.1 \pm 0.6 \\
5.2 \pm 0.5\end{array}$ & $\begin{array}{l}4.8 \pm 0.4 \\
5.3 \pm 0.4\end{array}$ \\
\hline $16: 1 \mathrm{n}-7$ trans & $\begin{array}{l}\text { Visit } 1 \\
\text { Visit } 2\end{array}$ & $\begin{array}{l}0.30 \pm 0.06 \\
0.23 \pm 0.04\end{array}$ & $\begin{array}{l}0.21 \pm 0.05 \\
0.25 \pm 0.02\end{array}$ \\
\hline 18:0 & $\begin{array}{l}\text { Visit } 1 \\
\text { Visit } 2\end{array}$ & $\begin{array}{l}1.6 \pm 0.1 \\
2.1 . \pm 0.6\end{array}$ & $\begin{array}{l}2.0 \pm 0.4 \\
1.5 \pm 0.2\end{array}$ \\
\hline $18: 1 \mathrm{n}-7$ trans & $\begin{array}{l}\text { Visit } 1 \\
\text { Visit } 2\end{array}$ & $\begin{array}{l}1.6 \pm 0.2 \\
1.4 \pm 0.2\end{array}$ & $\begin{array}{l}1.5 \pm 0.1 \\
1.7 \pm 0.4\end{array}$ \\
\hline $18: 1 \mathrm{n}-9$ cis & $\begin{array}{l}\text { Visit } 1 \\
\text { Visit } 2\end{array}$ & $\begin{array}{l}38.6 \pm 0.9 \\
34.3 \pm 0.9\end{array}$ & $\begin{array}{l}38.9 \pm 2.3 \\
34.4 \pm 1.3\end{array}$ \\
\hline $18: 1 \mathrm{n}-9$ trans & $\begin{array}{l}\text { Visit } 1 \\
\text { Visit } 2\end{array}$ & $\begin{array}{l}1.6 \pm 0.2 \\
1.4 \pm 0.2\end{array}$ & $\begin{array}{l}1.8 \pm 0.5 \\
1.6 \pm 0.4\end{array}$ \\
\hline $18: 2 n-6$ cis cis & $\begin{array}{l}\text { Visit } 1 \\
\text { Visit } 2\end{array}$ & $\begin{array}{l}21.0 \pm 0.8 \\
20.1 \pm 0.8\end{array}$ & $\begin{array}{l}23.5 \pm 1.7 \\
20.4 \pm 1.2\end{array}$ \\
\hline $\begin{array}{l}18: 2 \mathrm{n}-6 \text { trans } \\
\text { trans }\end{array}$ & $\begin{array}{l}\text { Visit } 1 \\
\text { Visit } 2\end{array}$ & $\begin{array}{l}1.8 \pm 0.2 \\
1.6 \pm 0.1\end{array}$ & $\begin{array}{l}2.1 \pm 0.2 \\
1.8 \pm 0.2\end{array}$ \\
\hline $18: 3 n-3$ all cis & $\begin{array}{l}\text { Visit } 1 \\
\text { Visit } 2\end{array}$ & $\begin{array}{l}2.9 \pm 0.2 \\
2.8 \pm 0.2\end{array}$ & $\begin{array}{l}2.9 \pm 0.4 \\
2.4 \pm 0.4\end{array}$ \\
\hline $20: 4 n-6$ all cis & $\begin{array}{l}\text { Visit } 1 \\
\text { Visit } 2\end{array}$ & $\begin{array}{l}1.8 \pm 0.1 \\
2.0 \pm 0.1\end{array}$ & $\begin{array}{l}2.5 \pm 0.2 \\
2.4 \pm 0.4\end{array}$ \\
\hline $20: 5 n-3$ all cis & $\begin{array}{l}\text { Visit } 1 \\
\text { Visit } 2\end{array}$ & $\begin{array}{l}0.13 \pm 0.02 \\
0.09 \pm 0.01\end{array}$ & $\begin{array}{l}0.13 \pm 0.02 \\
0.14 \pm 0.02\end{array}$ \\
\hline $22: 6 n-3$ all cis & $\begin{array}{l}\text { Visit } 1 \\
\text { Visit } 2\end{array}$ & $\begin{array}{l}1.7 \pm 0.5 \\
1.3 \pm 0.1\end{array}$ & $\begin{array}{l}1.8 \pm 0.4 \\
1.8 \pm 0.1\end{array}$ \\
\hline
\end{tabular}

Table 4

Serum individual free fatty acid mol \% correlations with HOMA-IR and HOMA-\% $\beta$ (average of visits 1 and 2)

\begin{tabular}{lcc}
\hline Fatty acid & HOMA-IR & HOMA $-\% \beta$ \\
\hline $12: 0$ & $-0.041(\mathrm{p}=0.883)$ & $0.102(\mathrm{p}=0.600)$ \\
$14: 0$ & $0.338(\mathrm{p}=0.073)$ & $0.198(\mathrm{p}=0.303)$ \\
$14: 1 \mathrm{n}-5$ cis & $0.050(\mathrm{p}=0.795)$ & $0.231(\mathrm{p}=0.227)$ \\
$14: 1 \mathrm{n}-5$ trans & $0.265(\mathrm{p}=0.165)$ & $0.145(\mathrm{p}=0.453)$ \\
$16: 0$ & $0.409(\mathrm{p}=0.027)$ & $0.222(\mathrm{p}=0.247)$ \\
$16: 1 \mathrm{n}-7$ cis & $0.181(\mathrm{p}=0.349)$ & $0.228(\mathrm{p}=0.234)$ \\
$16: 1 \mathrm{n}-7$ trans & $0.047(\mathrm{p}=0.807)$ & $-0.149(\mathrm{p}=0.439)$ \\
$18: 0$ & $0.282(\mathrm{p}=0.138)$ & $-0.054(\mathrm{p}=0.781)$ \\
$18: 1 \mathrm{n}-7$ trans & $-0.107(\mathrm{p}=0.672)$ & $-0.126(\mathrm{p}=0.619)$ \\
$18: 1 \mathrm{n}-9$ cis & $0.156(\mathrm{p}=0.420)$ & $0.123(\mathrm{p}=0.526)$ \\
$18: 1 \mathrm{n}-9$ trans & $-0.047(\mathrm{p}=0.811)$ & $0.240(\mathrm{p}=0.209)$ \\
$18: 2 \mathrm{n}-6$ cis cis & $-0.171(\mathrm{p}=0.375)$ & $-0.209(\mathrm{p}=0.278)$ \\
$18: 2 \mathrm{n}-6$ trans trans & $0.099(\mathrm{p}=0.610)$ & $0.227(\mathrm{p}=0.235)$ \\
$18: 3 \mathrm{n}-3$ all cis & $0.125(\mathrm{p}=0.519)$ & $-0.028(\mathrm{p}=0.885)$ \\
\hline $20: 4 \mathrm{n}-6$ all cis & $0.026(\mathrm{p}=0.895)$ & $-0.005(\mathrm{p}=0.980)$ \\
\hline $20: 5 \mathrm{n}-3$ all cis & $-0.179(\mathrm{p}=0.352)$ & $-0.200(\mathrm{p}=0.298)$ \\
\hline $22: 6 \mathrm{n}-3$ all cis & $-0.309(\mathrm{p}=0.103)$ & $-0.252(\mathrm{p}=0.187)$ \\
\hline
\end{tabular}

Table 5

Serum individual free fatty acid mol \% level correlations with HOMA-IR and HOMA-\% $\beta$ (visit 3 - flaxseed oil)

\begin{tabular}{lcc}
\hline Fatty acid & HOMA-IR & HOMA $-\% \beta$ \\
\hline $12: 0$ & $0.173(\mathrm{p}=0.508)$ & $0.304(\mathrm{p}=0.236)$ \\
$14: 0$ & $0.246(\mathrm{p}=0.341)$ & $0.172(\mathrm{p}=0.509)$ \\
$14: 1 \mathrm{n}-5$ cis & $0.502(\mathrm{p}=0.040)$ & $0.328(\mathrm{p}=0.199)$ \\
$14: 1 \mathrm{n}-5$ trans & $-0.234(\mathrm{p}=0.367)$ & $-0.135(\mathrm{p}=0.606)$ \\
$16: 0$ & $-0.086(\mathrm{p}=0.742)$ & $-0.190(\mathrm{p}=0.466)$ \\
$16: 1 \mathrm{n}-7$ cis & $0.000(\mathrm{p}=0.999)$ & $0.206(\mathrm{p}=0.428)$ \\
$16: 1 \mathrm{n}-7$ trans & $-0.359(\mathrm{p}=0.157)$ & $-0.360(\mathrm{p}=0.156)$ \\
$18: 0$ & $0.077(\mathrm{p}=0.769)$ & $-0.018(\mathrm{p}=0.944)$ \\
$18: 1 \mathrm{n}-7$ trans & $-0.149(\mathrm{p}=0.750)$ & $-0.339(\mathrm{p}=0.457)$ \\
$18: 1 \mathrm{n}-9$ cis & $-0.276(\mathrm{p}=0.284)$ & $-0.296(\mathrm{p}=0.248)$ \\
$18: 1 \mathrm{n}-9$ trans & $-0.273(\mathrm{p}=0.307)$ & $-0.403(\mathrm{p}=0.122)$ \\
$18: 2 \mathrm{n}-6$ cis cis & $-0.278(\mathrm{p}=0.281)$ & $-0.195(\mathrm{p}=0.454)$ \\
$18: 2 \mathrm{n}-6$ trans trans & $0.095(\mathrm{p}=0.716)$ & $0.248(\mathrm{p}=0.337)$ \\
$18: 3 \mathrm{n}-3$ all cis & $-0.522(\mathrm{p}=0.022)$ & $-0.442(\mathrm{p}=0.075)$ \\
\hline $20: 4 \mathrm{n}-6$ all cis & $-0.244(\mathrm{p}=0.346)$ & $-0.325(\mathrm{p}=0.203)$ \\
\hline $20: 5 \mathrm{n}-3$ all cis & $-0.090(\mathrm{p}=0.732)$ & $0.011(\mathrm{p}=0.966)$ \\
\hline $22: 6 \mathrm{n}-3$ all cis & $-0.345(\mathrm{p}=0.175)$ & $-0.226(\mathrm{p}=0.384)$ \\
\hline
\end{tabular}


Table 6

Serum individual free fatty acid mol \% level correlations with HOMA-IR and HOMA - $\% \beta$ (visit 3 - safflower oil)

\begin{tabular}{lcc}
\hline Fatty acid & HOMA-IR & HOMA $-\% \beta$ \\
\hline $12: 0$ & $0.000(\mathrm{p}=1.000)$ & $0.053(\mathrm{p}=0.878)$ \\
$14: 0$ & $-0.351(\mathrm{p}=0.289)$ & $0.219(\mathrm{p}=0.517)$ \\
$14: 1 \mathrm{n}-5$ cis & $-0.441(\mathrm{p}=0.175)$ & $0.266(\mathrm{p}=0.428)$ \\
$14: 1 \mathrm{n}-5$ trans & $0.180(\mathrm{p}=0.597)$ & $-0.073(\mathrm{p}=0.830)$ \\
$16: 0$ & $0.687(\mathrm{p}=0.019)$ & $-0.346(\mathrm{p}=0.297)$ \\
$16: 1 \mathrm{n}-7$ cis & $-0.117(\mathrm{p}=0.732)$ & $0.358(\mathrm{p}=0.280)$ \\
$16: 1 \mathrm{n}-7$ trans & $-0.014(\mathrm{p}=0.968)$ & $-0.241(\mathrm{p}=0.475)$ \\
$18: 0$ & $-0.481(\mathrm{p}=0.134)$ & $-0.537(\mathrm{p}=0.088)$ \\
$18: 1 \mathrm{n}-7$ trans & $0.475(\mathrm{p}=0.148)$ & $-0.221(\mathrm{p}=0.722)$ \\
$18: 1 \mathrm{n}-9$ cis & $-0.300(\mathrm{p}=0.370)$ & $-0.027(\mathrm{p}=0.938)$ \\
$18: 1 \mathrm{n}-9$ trans & $0.308(\mathrm{p}=0.357)$ & $-0.080(\mathrm{p}=0.815)$ \\
$18: 2 \mathrm{n}-6$ cis cis & $-0.262(\mathrm{p}=0.436)$ & $0.378(\mathrm{p}=0.252)$ \\
$18: 2 \mathrm{n}-6$ trans trans & $0.266(\mathrm{p}=0.430)$ & $0.357(\mathrm{p}=0.281)$ \\
$18: 3 \mathrm{n}-3$ all cis & $0.391(\mathrm{p}=0.234)$ & $0.312(\mathrm{p}=0.350)$ \\
\hline $20: 4 \mathrm{n}-6$ all cis & $-0.439(\mathrm{p}=0.177)$ & $0.116(\mathrm{p}=0.733)$ \\
\hline $20: 5 \mathrm{n}-3$ all cis & $-0.536(\mathrm{p}=0.089)$ & $0.189(\mathrm{p}=0.578)$ \\
\hline $22: 6 \mathrm{n}-3$ all cis & $0.302(\mathrm{p}=0.367)$ & $-0.139(\mathrm{p}=0.684)$ \\
\hline
\end{tabular}

tive correlation between each of myristic acid (MA, 14:0), myristelaidic acid (MEA, 14:1 n-5 trans), SA and HOMA-IR. There were no statistically significant correlations (though a negative trend with DHA) between the individual serum FFAs mol \% levels and each of HOMA-IR or HOMA- $\% \beta$. Flaxseed oil consumption eliminated the statistically significant positive correlation between PA and HOMA-IR found pre-supplementation, while at the same time inducing a statistically significant negative correlation between ALA and HOMA-IR and statistically significant positive correlation between myristoleic acid (MOA, 14:1 n-5) and HOMA-IR at visit 3 in flaxseed oil consumers only (Table 5 ).

Trends toward negative correlations between each of palmitelaidic acid (PEA, 16:1 n-7 trans), DHA and HOMA-IR were observed after flaxseed oil supplementation (Table 5). Trends toward a positive correlation between myristoleic acid (MOA, 14:1 n-5 cis) and HOMA- $\% \beta$ and negative correlations between each of PEA, elaidic acid (EA, 18:1 n-9 trans), ALA and HOMA- $\% \beta$ occurred in flaxseed oil consumers (Table 5).

Safflower oil consumers maintained the strong statistically significant positive correlation between
PA and HOMA-IR seen pre-supplementation while showing a positive trend with trans-vaccenic acid (TVA, 18:n-7 trans) and HOMA-IR with negative trend correlations between each of myristoleic acid (MOA, 14:1 n-5 cis), SA, AA, EPA and HOMA-IR (Table 6). Safflower oil consumers also showed a negative trend correlation between SA and HOMA- $\% \beta$ (Table 6).

MA, PO, LA mol \% levels dropped significantly while ALA and EPA rose significantly relative to baseline as the result of flaxseed oil supplementation (Table 7). Safflower oil produced no changes in mol $\%$ FFA (Table 7). There was no statistically significant difference between flaxseed oil and safflower oil treatment in going from pre-treatment (average of visits 1 and 2) to post treatment (visit 3) in terms of glucose, insulin, HOMA-IR and HOMA-\% $\beta$ (Table 8).

\section{Discussion}

Baseline FFA composition was similar to a previous report on serum FFA composition in T2D (Yang et al 2004). Individual FFAs pre-supplementation showed only a significant positive correlation between PA and HOMA-IR with no significant correlations between any of the fatty acids and HOMA- $\% \beta$. The data of Stefan et al. (2010) have suggested a role for decreasing HOMA-IR via palmitoleate (PO, 16:1 n-7 cis) but this was not demonstrated by the data in the current study (Tables 4-8). In the review, De Caterina et al. (2007) have noted that both the serum levels of PA and PO appeared to be inversely related to insulin sensitivity. The PA finding has consistency with the current study's finding of a positive significant correlation between PA and HOMA-IR in the pre-supplementation and safflower supplementation interventions.

Maris et al. (2011) suggested that oleate reduced $\beta$-cell function in vitro, a finding inconsistent with the current study. It is not clear from that paper that what type of oleate was used and whether that or any oleate might induce the same effect as OA in vivo (Tables $4-8$ ). Other in vitro work has suggested a decrease in the insulin release by $\beta$-cell exposed to PA and OA (Maris et al. 2011). Fontes et al (2009) have shown the same response for PA (our work did not bear this out). The work of Oprescu et al. (2007) in rat islets showing a decreased insulin secretion resulting from the incubation with $\mathrm{PA}$ and $\mathrm{OA}$ is not consistent with the current study. In contrast, PA has been suggested to increase insulin release (Parker et al. 2003) in rat islets and in contrast, reduce preproinsulin synthesis (insulin gene activity) and 
Table 7

$\mathrm{Mol} \%$ blood serum free fatty acid composition (mol\% levels) before and after flaxseed oil and safflower oil supplementation

\begin{tabular}{lccc}
\hline Fatty acid & $\begin{array}{c}\text { Visit } \mathbf{1} / \mathbf{2} \text { average } \\
\text { (pre-flaxseed and -safflower oil) }\end{array}$ & $\begin{array}{c}\text { Visit } \mathbf{3} \\
\text { (post-flaxseed oil) }\end{array}$ & $\begin{array}{c}\text { Visit } 3 \\
\text { (post-safflower oil) }\end{array}$ \\
\hline $12: 0$ & $1.0 \pm 0.1$ & $0.91 \pm 0.18$ & $1.0 \pm 0.2$ \\
$14: 0$ & $2.8 \pm 0.1$ & $2.3 \pm 0.3$ & $2.6 \pm 0.1$ \\
$14: 1 \mathrm{n}-5$ cis & $0.11 \pm 0.01$ & $0.21 \pm 0.05$ & $0.15 \pm 0.02$ \\
$14: 1 \mathrm{n}-5$ trans & $0.14 \pm 0.01$ & $0.12 \pm 0.01$ & $0.15 \pm 0.01$ \\
$16: 0$ & $21.7 \pm 0.5$ & $22.9 \pm 0.4$ & $21.4 \pm 0.6$ \\
$16: 1 \mathrm{n}-7$ cis & $5.5 \pm 0.2$ & $4.4 \pm 0.5$ & $5.3 \pm 0.3$ \\
$16: 1 \mathrm{n}-7$ trans & $0.23 \pm 0.01$ & $\mathrm{p}=0.019$ vs. baseline & $0.27 \pm 0.01$ \\
$18: 0$ & $1.7 \pm 0.2$ & $0.30 \pm 0.04$ & $1.7 \pm 0.3$ \\
$18: 1 \mathrm{n}-7$ trans & $1.3 \pm 0.1$ & $1.7 \pm 0.3$ & $1.9 \pm 0.1$ \\
$18: 1 \mathrm{n}-9$ cis & $36.3 \pm 0.7$ & $1.6 \pm 0.3$ & $36.5 \pm 2.5$ \\
$18: 1 \mathrm{n}-9$ trans & $1.5 \pm 0.1$ & $37.7 \pm 1.7$ & $1.9 \pm 0.4$ \\
$18: 2 \mathrm{n}-6$ cis cis & $22.5 \pm 0.6$ & $1.6 \pm 0.3$ & $23.1 \pm 1.0$ \\
\hline $18: 2 \mathrm{n}-6$ trans trans & $1.7 \pm 0.1$ & $19.9 \pm 1.4$ & $1.9 \pm 0.5$ \\
\hline $18: 3 \mathrm{n}-3$ all cis & $2.8 \pm 0.1$ & $\mathrm{p}=0.019$ vs. baseline & $2.9 \pm 0.2$ \\
\hline $20: 4 \mathrm{n}-6$ all cis & $2.2 \pm 0.1$ & $1.3 \pm 0.1$ & $2.0 \pm 0.2$ \\
\hline $20: 5 \mathrm{n}-3$ all cis & $0.11 \pm 0.01$ & $4.8 \pm 0.4$ & $0.12 \pm 0.02$ \\
\hline $2: 6 \mathrm{n}-3$ all cis & $1.5 \pm 0.1$ & $\mathrm{p}=0.035$ vs. baseline and safflower oil & $1.3 \pm 0.2$ \\
\hline
\end{tabular}

perhaps by extension insulin and hence HOMA- $\% \beta$ (Kelpe et al. 2003) though this did not manifest in a change in correlations seen in the current study (Tables 4-6).

It seems that FFAs are more correlative preand post-supplementation with HOMA-IR than HOMA- $\% \beta$ though the significance of this observation is not clear. Trends in correlations did not affect the outcomes to supplementation in terms of HOMA-IR and HOMA- $\% \beta$.

Muramatsu et al. (2010) have shown an association of increased dietary intake of ALA with reduced insulin resistance in healthy persons, although their association was not correlative but rather assessed in terms of lower absolute levels of HOMA-IR. This relationship is consistent with the statistically significant negative correlation between ALA and HOMA-IR upon flaxseed oil supplementation seen in the current study. Wang et al. (2013) have found that feeding pure ALA decreased HOMA-IR in obese (not T2Ds) persons
Table 8

Glucose, insulin HOMA-IR and HOMA- $\% \beta$ values before and after flaxseed oil and safflower oil supplementation

\begin{tabular}{lccc}
\hline Parameter & $\begin{array}{c}\text { Visit 1 and 2 } \\
\text { (average) }\end{array}$ & $\begin{array}{c}\text { Visit 3 } \\
\text { FXO }\end{array}$ & $\begin{array}{c}\text { Visit 3 } \\
\text { SFO }\end{array}$ \\
\hline $\mathrm{N}$ & 18 & 18 & 14 \\
$\begin{array}{l}\mathrm{BMI}\left(\mathrm{kg} / \mathrm{m}^{2}\right) \\
\mathrm{p}=0.85\end{array}$ & $31.3 \pm 0.9$ & $33.0 \pm 1.4$ & $30.8 \pm 1.2$ \\
$\begin{array}{l}\text { Glucose }(\mathrm{mmol} / \mathrm{l}) \\
\mathrm{p}=0.06\end{array}$ & $8.0 \pm 0.8$ & $9.0 \pm 0.9$ & $8.5 \pm 1.2$ \\
$\begin{array}{l}\text { Insulin }(\mathrm{mU} / \mathrm{ml}) \\
\mathrm{p}=0.07\end{array}$ & $10.5 \pm 2.6$ & $17.7 \pm 5.2$ & $7.0 \pm 1.8$ \\
$\begin{array}{l}\text { HOMA-IR } \\
\mathrm{p}=0.17\end{array}$ & $3.7 \pm 0.5$ & $7.1 \pm 2.4$ & $2.6 \pm 0.6$ \\
$\begin{array}{l}\text { HOMA-\% } \beta \\
\mathrm{p}=0.08\end{array}$ & $46.9 \pm 9.0$ & $54.4 \pm 14.8$ & $28.0 \pm 5.6$ \\
\hline
\end{tabular}

A general linear model analysis (repeated measures) was performed with a significance level of $\mathrm{p}<0.05$.

FXO - flaxseed oil; SFO - safflower oil 
again consistent with the negative correlation seen in the current study. Nonetheless, the significant negative correlation seen in our study between mol \% serum free ALA levels and HOMA-IR, and the significant increase in mol \% levels of serum free ALA (Table 7) is consistent with a contribution to improved HOMA-IR or glycemic control in general (albeit overcome by indeterminate factors in our study and other works with flaxseed oil) (Barre et al. 2008; Foster et al. 2013; Taylor et al. 2010). Consequently, one might suggest that it is the delivery (pure ALA versus ALA in oil format) that determines whether ALA reduces HOMA-IR. The lower ALA mol \% levels seen in the study of Yi et al. (2007) in T2Ds are consistent with the negative correlation seen in the current study (Table 5).

Unlike the work of Asp et al. (2011) with safflower oil, the current study (Table 6) showed no change in the glycemic control despite safflower oil induced trends in some FA relationships with HOMA-IR and HOMA- $\% \beta$ not seen in the pre-treatment/placebo data (Table 4).

Despite the significant changes in blood serum mol \% of some individual FFAs as the result of flaxseed oil or safflower administration (Table 7), none of the FFAs correlated significantly with HOMA- $\% \beta$ before or after the flaxseed or safflower oil consumption. It is consistent with the finding that fish oil rich in EPA and DHA did not influence HOMA-IR in T2Ds or the obese (Crochemore et al. 2012) (in flaxseed oil consumers, EPA and DHA can be derived from ALA). Various authors have observed either an increase or no impact on the blood plasma EPA levels and no impact on DHA levels as the result of ALA consumption in various oil formats (Ezaki et al. 1996; Hamazaki et al. 2006; Plourde and Cunnane 2007). However, the current results reflect a specific oil (flaxseed oil) and a specific pool (serum FFA) and so a comparison between papers using different oils and different blood plasma FA pools (e.g. phospholipid, total) is difficult.

Any individual statistically significant correlation or trend toward such between a given FA level and each of HOMA-IR and HOMA- $\% \beta$ explained a relatively low contribution $\left(\mathrm{r}^{2}\right.$ value) to the variability of HOMA-IR and HOMA-\% $\beta$ by that FA. Indeed, there was no change in the HOMA-IR and HOMA- $\% \beta$ as the result of flaxseed oil compared to safflower oil administration (Table 8) consistent in terms of HOMA-IR findings (Eyjolfson et al. 2004; Taylor et al. 2010). There appear to be no published studies on the impact of ALA or flaxseed oil on HOMA-\% $\beta$.
Flaxseed oil consumption did not alter glucose, insulin, and HOMA-IR and/or HOMA- $\% \beta$ relative to placebo (safflower oil) (Table 8). However, a larger study may demonstrate changes in one or more of these parameters for flaxseed oil versus safflower oil. While mol \% individual FFAs and correlations between various serum individual FFA mol \% levels and HOMA-IR and HOMA- $\% \beta$ can be manipulated significantly by flaxseed oil, correlations arising from this supplementation, do not result in a shift in glucose, insulin, HOMA-IR and/or HOMA- $\% \beta$ despite changes in mol \% FFAs induced by flaxseed oil (Tables 4-8). The absence of change in glucose, insulin, and HOMA-IR and/or HOMA- $\% \beta$ may be due to a flaxseed oil generated mix of negative and positive correlations cancelling one another out (Tables 4-6). It may be that feeding or perhaps infusing pure ALA will not only result in a negative correlation between HOMA-IR and ALA, but also in a statistically significant HOMA-IR decrease with an, as yet indeterminate, impact on HOMA- $\% \beta$. Flaxseed oil is mix of FAs each of which may have differing impacts on the correlations. However, this work shows that there are correlations and/or trends towards statistically significant correlations between some serum individual free fatty acids and HOMAIR and HOMA- $\% \beta$. The findings of the current study could not be influenced by unchanged patterns in diet, smoking, exercise or medications (dose/type) over the course of the study.

The significance of this study is perhaps that the delivery platform (e.g. flaxseed oil versus pure ALA) of dietary FAs that influences whether a particular serum FFA can influence HOMA-IR and/or HOMA- $\% \beta$ and thus perhaps produce better clinical outcomes to the extent that diet may contribute to such. However, this remains to be further investigated. It also remains to investigate this study design using a large number of patients to address the limitation of the small number of patients in this study, HOMA-IR and/or HOMA- $\% \beta$ may be inaccurate or imprecise.

This is the first publication, in which we are aware that different relationships (correlations) and/or trends towards statistically significant correlations between mol \% levels of serum individual FFAs each of HOMA-IR and HOMA- $\% \beta$ in human T2D are shown and that these correlations can be manipulated by the administration of flaxseed oil and safflower oil.

In conclusion, the primary hypothesis that there would be different relationships (correlations) between blood serum individual FFAs mol \% levels and each of HOMA-IR and/or HOMA- $\% \beta$ in human 
T2D is supported by the data. The secondary hypothesis that flaxseed oil (rich in ALA) supplementation in comparison to the placebo safflower oil (poor in ALA) would alter these correlations is supported. However, there were no alterations in HOMA-IR and/ or HOMA- $\% \beta$ and consequently it is concluded that changes in these correlations did not lead to changes in HOMA-IR and/or HOMA-\% $\beta$. However, a larger study with flaxseed oil or with a delivery platform for ALA other than flaxseed oil might reveal statistically significant changes in these variables.

\section{Acknowledgements}

This work was supported by the Cape Breton University Research Assistance Programme and Summer Stipend Research Assistance grants for operating funds, Canadian Institutes for Health Research institutional grant (to Cape Breton University) for operating funds, Canada Foundation for Innovation and Nova Scotia Health Research Foundation for equipment grants. The authors would like to thank Ms. Emily Stelmach, B.Sc. for the data analyses.

\section{References}

Armoni M, Harel C, Bar-Yoseph F, Milo S, Karnieli E. Free fatty acids repress the GLUT4 gene expression in cardiac muscle via novel response elements. J Biol Chem 280, 34786-34795. 2005.

Asp ML, Collene AL, Norris LE, Cole RM, Stout MB, Tang SY, Hsu JC, Belury MA. Time-dependent effects of safflower oil to improve glycemia, inflammation and blood lipids in obese, post-menopausal women with type 2 diabetes, a randomized, double-masked, crossover study. Clin Nutr 30, 443-449, 2011.

Barre DE, Griscti O, Mizier-Barre KA, Hafez K. Flaxseed oil and lipoprotein (a) significantly increase bleeding time in type 2 diabetes patients in Cape Breton, Nova Scotia, Canada. J Oleo Sci 54, 347-354, 2005 a.

Barre DE, Griscti O, Mizier-Barre KA, Hafez K. The mechanism by which flaxseed oil consumption increases bleeding time in patients with type 2 diabetes in Cape Breton, Nova Scotia, Canada is independent of lipoprotein (a) concentration. J Oleo Sci 54, 617-625, 2005 b.

Barre DE, Mizier-Barre KA, Griscti O, Hafez K. High dose flaxseed oil supplementation may affect fasting blood serum glucose management in human type 2 diabetics. J Oleo Sci 57, 269-273, 2008.

Blaak EE. Fatty acid metabolism in obesity and type 2 diabetes mellitus. Proc Nutr Soc 62, 753-760, 2003.

Boden G. Obesity, insulin resistance and free fatty acids. Curr Opin Endocrinol Diabetes Obes 18, 139-143, 2011.

Butler TJ, Barriocanal LA, Walker M. Elevated plasma non-esterified fatty acid levels and insulin secretion in nondiabetic relatives of type 2 diabetic patients. Clin Endocrinol (Oxf) 55, 349-355, 2001.

Crochemore IC, Souza AF, de Souza AC, Rosado EL. $\omega$-3 polyunsaturated fatty acid supplementation does not influence body composition, insulin resistance, and lipemia in women with type 2 diabetes and obesity. Nutr Clin Pract 27, 553-560, 2012.

De Caterina R, Madonna R, Bertolotto A, Schmidt EB. n-3 fatty acids in the treatment of diabetic patients, biological rationale and clinical data. Diabetes Care 30, 1012-1026, 2007.

Delarue J, Magnan C. Free fatty acids and insulin resistance, Curr Opin Clin Nutr Metab Care 10, 142-148, 2007.

Eriksson J, Saloranta C, Widen E, Ekstrand A, Franssila-Kallunki A, Schalin C, Groop L. Non-esterified fatty acids do not contribute to insulin resistance in persons at increased risk of developing type 2 (non-insulin-dependent) diabetes mellitus. Diabetologia 34, 192-197, 1991.

Eyjolfson V, Spriet LL, Dyck DJ. Conjugated linoleic acid improves insulin sensitivity in young, sedentary humans. Med Sci Sports Exerc 36, 814-820, 2004.

Ezaki O, Takahashi M, Shigematsu T, Shimamura K, Kimura J, Ezaki H, Gotoh T. Long-term effects of dietary alphalinolenic acid from perilla oil on serum fatty acids composition and on the risk factors of coronary heart disease in Japanese elderly subjects. J Nutr Sci Vitaminol (Tokyo) 45, 759-772, 1996.

Fontes G, Semache M, Hagman DK, Tremblay C, Shah R, Rhodes CJ, Rutter J, Poitout V. Involvement of Per-ArntSim Kinase and extracellular-regulated kinases-1/2 in palmitate inhibition of insulin gene expression in pancreatic beta-cells. Diabetes 58, 2048-2058, 2009.

Foster M, Petocz P, Caterson ID, Samman S. Effects of zinc and $\alpha$-linolenic acid supplementation on glycemia and lipidemia in women with type 2 diabetes mellitus, a randomized, double-blind, placebo-controlled trial. Journal of Diabetes Research and Clinical Metabolism 2, 3, 2013.

Glauber H, Wallace P, Griver K, Brechtel G. Adverse metabolic effect of omega-3 fatty acids in non-insulin-dependent diabetes mellitus. Ann Intern Med 108, 663-668, 1998. 
Goh Y, Jumpsen J, Ryan E, Clandinin M. Effect of omega 3 fatty acid on plasma lipids, cholesterol and fatty acid content in NIDDM patients. Diabetologia 40, 45-52, 1997.

Grapov D, Adams SH, Pedersen TL, Garvey WT, Newman JW. Type 2 diabetes associated changes in the plasma nonesterified fatty acids, oxylipins and endocannabinoids. PLoS One 7, e48852, 2012.

Hamazaki K, Itomura M, Hamazaki T, Sawazaki S. Effects of cooking plant oils on recurrent aphthous stomatitis, a randomized, placebo-controlled, double-blind trial. Nutrition 22, 534-538, 2006.

Karpe F, Dickmann JR, Frayn KN. Fatty acids, obesity, and insulin resistance, time for a reevaluation. Diabetes 10 , 2441-2449, 2011.

Kelpe CL, Moore PC, Parazzoli SD, Wicksteed B, Rhodes CJ, Poitout V. Palmitate inhibition of insulin gene expression is mediated at the transcriptional level via ceramide synthesis. J Biol Chem 278, 30015-30021, 2003.

Liu L, Li Y, Guan C, Li K, Wang C, Feng R, Sun C. Free fatty acid metabolic profile and biomarkers of isolated post-challenge diabetes and type 2 diabetes mellitus based on GC-MS and multivariate statistical analysis. J Chromatogr B Analyt Technol Biomed Life Sci 878, 2817-2825, 2010.

Hawkins M, Tonelli J, Kishore P, Stein D, Ragucci E, Gitig A, Reddy K. Contribution of Elevated Free Fatty Acid Levels to the Lack of Glucose Effectiveness in Type 2 Diabetes. Diabetes 52, 2748-2758, 2003.

Maris M, Waelkens E, Cnop M, D’Hertog W, Cunha DA, Korf H, Koike T, Overbergh L, Mathieu C. Oleate-induced beta cell dysfunction and apoptosis, a proteomic approach to glucolipotoxicity by an unsaturated fatty acid. J Proteome Res 10, 3372-3385, 2011.

Matthews DR, Hosker JP, Rudenski AS, Naylor BA, Treacher DF, Turner RC. Homeostasis model assessment: insulin resistance and beta-cell function from fasting plasma glucose and insulin concentrations in man. Diabetologia 28, 412-419, 1985.

McManus R, Clandinin M, Jumpson J, Finegood DT, Clandinin MT, Ryan EA. A comparison of the effects of n-3 fatty acids from linseed oil and fish oil in well-controlled type 2 diabetes. Diabetes Care 19, 463-467, 1996.

Mehta A, Oeser AM, Carlson MG. Rapid quantitation of free fatty acids in human plasma by high-performance liquid chromatography. J Chromatogr B Biomed Sci Appl 719, 9-23, 1998.

Montori V, Wollan P, Farmer A, Dinneen S. Fish oil supplementation in type 2 diabetes. Diabetes Care 23, 1407-1415, 2000.

Moore PC, Ugas MA, Hagman DK, Parazzoli SD, Poitout V. Evidence against the involvement of oxidative stress in fatty acid inhibition of insulin secretion. Diabetes 53, 2610-2616, 2004.

Morita S, Shimajiri Y, Sakagashira S, Furuta M, Sanke T. Effect of exposure to non-esterified fatty acid on progressive deterioration of insulin secretion in patients with Type 2 diabetes, a long-term follow-up study. Diabet Med 29, 980-985, 2012.

Muramatsu T, Yatsuya H, Toyoshima H, Sasaki S, Li Y, Otsuka R, Wada K, Hotta Y, Mitsuhashi H, Matsushita K, Murohara T, Tamakoshi K. Higher dietary intake of alpha-linolenic acid is associated with lower insulin resistance in middle-aged Japanese. Prev Med 50, 272-276. 2010.

Nakamura N, Hamazaki T, Kobayashi M, Ohta M, Okuda K. Effects of eicosapentaenoic acids on remnant like particles, cholesterol concentrations and plasma fatty acid composition in patients with diabetes mellitus. In vivo 12, 311-314, 1998.

Nettleton J, Katz R. n-3 long-chain polyunsaturated fatty acids in type 2 diabetes. J Am Dietetic Assoc 105, 428-440, 2005.

Newens KJ, Thompson AK, Jackson KG, Wright J, Williams CM. Acute effects of elevated NEFA on vascular function, a comparison of SFA and MUFA. Br J Nutr 105, 1343-1351, 2011.

Wang DQ, Liu XL, Rong QF, Han L, Zhao NQ. Alpha-linolenic acid improves insulin sensitivity in obese patients. Zhonghua Yi Xue Za Zhi 93, 132-134, 2013.

Oprescu AI, Bikopoulos G, Naassan A, Allister EM, Tang C, Park E, Uchino H, Lewis GF, Fantus IG, Rozakis-Adcock M, Wheeler MB, Giacca A. Free fatty acid-induced reduction in glucose-stimulated insulin secretion, evidence for a role of oxidative stress in vitro and in vivo. Diabetes 56, 2927-2937, 2007.

Parker SM, Moore PC, Johnson LM, Poitout V. Palmitate potentiation of glucose-induced insulin release, a study using 2-bromopalmitate. Metabolism 52, 1367-1371, 2003.

Plourde M, Cunnane SC. Extremely limited synthesis of long chain polyunsaturates in adults, implications for their dietary essentiality and use as supplements. Appl Physiol Nutr Metab 32, 619-634, 2007.

Poitout V, Hagman D, Stein R, Artner I, Robertson RP, Harmon JS. Regulation of the insulin gene by glucose and fatty acids. J Nutr 136, 873-876, 2006.

Poitout V, Robertson RP. Glucolipotoxicity, fuel excess and beta-cell dysfunction. Endocr Rev 29, 351-366, 2008. 
Poitout V, Amyot J, Semache M, Zarrouki B, Hagman D, Fontes G. Glucolipotoxicity of the pancreatic beta cell. Biochim Biophys Acta 1801, 289-229, 2010.

Reaven GM, Chen Y. Role of abnormal free fatty acid metabolism in the development of non-insulin-dependent diabetes mellitus. Am J Med 85, 106-112, 1988.

Rivellese AA, Maffettone A, Iovine C, Di Marino L, Annuzzi G, Mancini M, Riccardi G. Long-term effects of fish oil on insulin resistance and plasma lipoproteins in NIDDM patients with hypertriglyceridemia. Diabetes Care 19, 1207-1213, 1996.

Robertson RP, Harmon J, Tran PO, Poitout V. Beta-cell glucose toxicity, lipotoxicity, and chronic oxidative stress in type 2 diabetes. Diabetes 53, 119-124, 2004.

Roscoe JT. Fundamental Research Statistics for the Behavioral Sciences. Second Edition. Holt, Reinhart and Winston, Montreal 217, 1975.

Salgin B, Ong KK, Thankamony A, Emmett P, Wareham NJ, Dunger DB. Higher fasting plasma free fatty acid levels are associated with lower insulin secretion in children and adults and a higher incidence of type 2 diabetes. J Clin Endocrinol Metab 97, 302-309, 2012.

Stefan N, Kantartzis K, Celebi N, Staiger H, Machann J, Schick F, Cegan A, Elcnerova M, Schleicher E, Fritsche A, Haring HU. Circulating palmitoleate strongly and independently predicts insulin sensitivity in humans. Diabetes Care 33, 405-407, 2010.

Taylor CG, Noto AD, Stringer DM, Froese S, Malcolmson L. Dietary milled flaxseed and flaxseed oil improve N-3 fatty acid status and do not affect glycemic control in individuals with well-controlled type 2 diabetes. J Am Coll Nutr 29, 72-80, 2010.

Wallace TM, Levy JC, Matthews DR. Use and abuse of HOMA modeling. Diabetes Care 27, 1487-1495, 2004.

Woodman R, Mori T, Burke V, Puddey IB, Watts GF, Beilin LJ. Effects of purified eicosapentaenoic and docosahexaenoic acids on glycemic control, blood pressure and serum lipids in type 2 diabetic patients with treated hypertension. Am J Clin Nutr 76, 1007-1015, 2002.

Yang J, Xu G, Hong Q, Liebich HM, Lutz K, Schmulling RM, Wahl HG. Discrimination of Type 2 diabetic patients from healthy controls by using metabonomics method based on their serum fatty acid profiles. J Chromatogr B Analyt Technol Biomed Life Sci 813, 53-58, 2004.

Ye J. Role of insulin in the pathogenesis of free fatty acid-induced insulin resistance in skeletal muscle. Endocr Metab Immune Disord Drug Targets 7, 65-74, 2007.

Yi L, He J, Liang Y, Yuan D, Gao H, Zhou H. Simultaneously quantitative measurement of comprehensive profiles of esterified and non-esterified fatty acid in plasma of type 2 diabetic patients. Chem Phys Lipids 150, 204-216, 2007. 